\title{
Mesenchymal stem cells transplantation suppresses inflammatory responses in global cerebral ischemia: contribution of TNF- $\alpha$-induced protein 6
}

\author{
Qing-ming $\mathrm{LIN}^{1,2}$, Shen ZHAO ${ }^{1,2}$, Li-li ZHOU ${ }^{1,2}$, Xiang-shao FANG ${ }^{1,2}$, Yue FU ${ }^{1,2}$, Zi-tong HUANG ${ }^{1,2, *}$ \\ ${ }^{1}$ Department of Emergency, Sun Yat-sen Memorial Hospital, Sun Yat-sen University, Guangzhou 510120, China; ${ }^{2}$ Institute of Cardiopul- \\ monary Cerebral Resuscitation, Sun Yat-sen University, Guangzhou 510120, China
}

Aim: To investigate the effects of mesenchymal stem cells (MSCs) transplantation on rat global cerebral ischemia and the underlying mechanisms.

Methods: Adult male SD rats underwent asphxial cardiac arrest to induce global cerebral ischemia, then received intravenous injection of $5 \times 10^{6}$ cultured MSCs of SD rats at $2 \mathrm{~h}$ after resuscitation. In another group of cardiac arrest rats, tumor necrosis factor- $\alpha$-induced protein 6 (TSG-6, $6 \mu \mathrm{g}$ ) was injected into the right lateral ventricle. Functional outcome was assessed at 1, 3, and $7 \mathrm{~d}$ after resuscitation. Donor MSCs in the brains were detected at $3 \mathrm{~d}$ after resuscitation. The level of serum S-100B and proinflammatory cytokines in cerebral cortex were assayed using ELISA. The expression of TSG-6 and proinflammatory cytokines in cerebral cortex was assayed using RT-PCR. Western blot was performed to determine the levels of TSG-6 and neutrophil elastase in cerebral cortex.

Results: MSCs transplantation significantly reduced serum S-100B level, and improved neurological function after global cerebral ischemia compared to the PBS-treated group. The MSCs injected migrated into the ischemic brains, and were observed mainly in the cerebral cortex. Furthermore, MSCs transplantation significantly increased the expression of TSG-6, and reduced the expression of neutrophil elastase and proinflammatory cytokines in the cerebral cortex. Intracerebroventricular injection of TSG-6 reproduced the beneficial effects of MSCs transplantation in rats with global cerebral ischemia.

Conclusion: MSCs transplantation improves functional recovery and reduces inflammatory responses in rats with global cerebral ischemia, maybe via upregulation of TSG-6 expression.

Keywords: mesenchymal stem cells; stem cells transplantation; cardiac arrest; global cerebral ischemia; tumor necrosis factor- $\alpha$ induced protein 6; inflammation; cytokine; neutrophil elastase

Acta Pharmacologica Sinica (2013) 34: 784-792; doi: 10.1038/aps.2012.199; published online 11 Mar 2013

\section{Introduction}

Cardiac arrest is the common cause of global cerebral ischemia, and has a low survival rate. In industrial countries, $14 \%$ to $40 \%$ of adult patients after an out-of-hospital cardiac arrest regained return of spontaneous circulation (ROSC) and were admitted to hospital ${ }^{[1,2]}$. Only $7 \%$ to $30 \%$ of resuscitated patients were discharged from hospital with a good neurological outcome ${ }^{[1]}$. Of those patients discharged from hospital, half of patients still had various forms of cognitive deficits ${ }^{[3]}$. Brain damage caused by ischemia/reperfusion injury after cardiopulmonary resuscitation (CPR) from cardiac arrest is marked by ischemic neuronal changes scattered throughout most brain regions ${ }^{[4]}$. Secondary inflammatory responses dur-

\footnotetext{
* To whom correspondence should be addressed.

E-mail huangztdoc@126.com

Received 2012-10-21 Accepted 2012-12-20
}

ing and after CPR are thought to be the mainly pathophysiologic mechanisms of brain damage ${ }^{[5]}$, suggesting therapeutic strategies aimed at preventing post-ischemic inflammation may improve functional recovery.

Bone marrow-derived mesenchymal stem cells (MSCs) are capable of differentiating into cells of the neuronal and glial lineage both in vivo and in vitro ${ }^{[6,7]}$. Since MSCs can be readily obtained from bone marrow, easily expanded in culture, and they are generally not tumorigenic, transplantation of MSCs has been used in the treatment of central nervous system injury including cerebral ischemia. In the model of focal cerebral ischemia (middle cerebral artery occlusion model), MSCs have been shown to reduce infarct size and improve neurological function ${ }^{[8-13]}$. Although few studies have been done in global cerebral ischemia, data also demonstrate functional improvement following systemic delivery of MSCs ${ }^{[6,14,15]}$. The potential mechanisms by which MSCs 
promote functional recovery include angiogenesis ${ }^{[8,9]}$, neurogenesis $^{[16,17]}$, neuroprotection ${ }^{[10,14]}$, expression of growth factors or cytokines ${ }^{[12,13]}$. However, the precise mechanisms of action of MSCs require further investigation.

In the present study, we hypothesized that intravenously injected MSCs after global cerebral ischemia induced by cardiac arrest suppressed post-ischemic inflammation in the brain possibly via an elevated anti-inflammatory factor and improved neurological function in rats. To test the hypothesis, we examined inflammatory markers, anti-inflammatory factor tumor necrosis factor-a-induced protein 6 (TSG-6) and neurological function in global cerebral ischemic rats following systematically MSCs transplantation or topically TSG-6 injection.

\section{Materials and methods}

The protocol of the experiment was approved by the Sun YatSen University Institutional Animal Ethic Committee. Fiveweek-old male healthy Sprague-Dawley (SD) rats weighing 100-150 g or ten-week-old male healthy SD rats weighing 300$400 \mathrm{~g}$ used in this experiment were bought from Experimental Animal Center of Sun Yat-Sen University [Grade II, Certification № SCXK (YUE) 2011-0029].

\section{Isolation and culture of MSCs derived from bone marrow}

MSCs derived from bone marrow as previously described ${ }^{[6]}$ were isolated and cultured. Briefly, the femur and tibia from five-week-old male SD rats were excised and bone marrow was obtained by washing the cavity with Dulbecco's modified Eagle's medium- $\mathrm{F}_{12}$ (DMEM-F $\mathrm{D}_{12} ;$ Gibco, USA). After collecting cell suspension, the cells were centrifuged at $310 \times g$ for 5 min. The cells were then resuspended in DMEM-F $\mathrm{F}_{12}$ with $10 \%$ fetal bovine serum (Hyclone, USA), $100 \mathrm{IU} / \mathrm{mL}$ penicillin, and $100 \mu \mathrm{g} / \mathrm{mL}$ streptomycin (Sigma, USA), plated in $25-\mathrm{cm}^{2}$ plastic culture flasks, and incubated in a humidified atmosphere of $5 \% \mathrm{CO}_{2}$ at $37^{\circ} \mathrm{C}$ for $3 \mathrm{~d}$. The medium was removed after $3 \mathrm{~d}$ and the adherent cells were cultured for 4 to $6 \mathrm{~d}$ until approximately $90 \%$ confluence was reached. These cells were trypsinized with $0.25 \%$ trypsin-EDTA (Gibco, USA) and subcultured at the ratio of 1:2. Passage two MSCs were expanded with the same protocol and passage three MSCs were used in all experiments. MSCs were identified with flow cytometry according to previously described methods ${ }^{[18]}$ and were negative for CD11b and CD45, and positive for CD29 (>95\%) and CD44 (>95\%). Passage three MSCs were labeled with 4',6'-diamidino-2-phenylindole (DAPI; Roche, USA) before administration as previously described ${ }^{[19]}$. Prior to transplantation, the cells were resuspended in $0.5 \mathrm{~mL}$ phosphate buffer solution (PBS) at a concentration of $10^{7}$ cells $/ \mathrm{mL}$ for injection.

\section{Animal model of global cerebral ischemia}

Global cerebral ischemia was induced by asphyxial cardiac arrest, as previously described ${ }^{[20]}$. In brief, after an overnight fast except for free access to water, male SD rats weighing 300-400 g were anesthetized by intraperitoneal injection of 45 $\mathrm{mg}$ per $\mathrm{kg}$ pentobarbital sodium. Additional doses of $10 \mathrm{mg}$ per $\mathrm{kg}$ were administered at intervals of approximately $1 \mathrm{~h}$ if necessary. The tracheal was orally intubated with a 14 gauge cannula (Abbocath-T, USA).

A 23 gauge polyethylene 50 (PE-50) catheter (Abbocath-T, USA) was advanced through the right external jugular vein into the right atrium for MSCs transplantation. Through the left femoral artery, a 23 gauge PE-50 catheter was advanced into the thoracic aorta for measurement of mean arterial pressure (MAP) and withdrawal of blood for arterial blood gas analysis. Another 23 gauge PE-50 catheter was also advanced through the left femoral vein into the inferior vena cava for administration of medications. MAP was measured with a pressure transducer (BD, Germany). Prior to insertion, the catheters were filled with physiological salt solution containing $5 \mathrm{IU} / \mathrm{mL}$ of heparin. Electrocardiogram lead II was recorded. Rectal temperature was maintained at $36.5 \pm 0.5^{\circ} \mathrm{C}$ with an incandescent heating lamp. The animals were mechanically ventilated with a fraction of inspired oxygen $\left(\mathrm{FiO}_{2}\right)$ of $21 \%$ at a tidal volume of $6.5 \mathrm{~mL} / \mathrm{kg}$ animal weight and a frequency of 100 breaths/min. Hemodynamic data were recorded in a six channel recorder (Windaq acquisition system, USA).

Asphyxia was induced by intravenous injection of $1 \mathrm{mg}$ per $\mathrm{kg}$ vecuronium and discontinuation of ventilation. Cardiac arrest was determined by loss of aortic pulsation, defined as MAP $\leq 20 \mathrm{mmHg}$, which occurred approximately 4 min after asphyxia. Six minutes after onset of cardiac arrest, precordial compression was initiated with an electrically driven mechanical chest compressor and mechanical ventilation with a $\mathrm{FiO}_{2}$ of $100 \%$ was resumed. Compression rate was maintained at a rate of $200 \mathrm{~min}^{-1}$ and synchronized with a compression/ventilation ratio of 2:1 with equal compression-relaxation duration. The depth of compression was adjusted to maintain an arterial diastolic pressure at $25 \pm 5 \mathrm{mmHg}$. ROSC was defined as return of a supraventricular rhythm with a MAP $\geq 60 \mathrm{mmHg}$ lasting for $\geq 5 \mathrm{~min}$. Precordial compression was stopped when there was no ROSC after $4 \mathrm{~min}$. Attempted resuscitation was discontinued after 4 min because the animals nearly survived for neurological functional testing in our prior study. Mechanical ventilation was continued for two additional hours after ROSC. $\mathrm{FiO}_{2}$ was $100 \%$ at $0-0.5 \mathrm{~h}$ after ROSC, $50 \%$ at $0.5-1 \mathrm{~h}$ and $30 \%$ at 1-2 h. Sham-operated rats underwent the same operation but without inducing cardiac arrest. When upper airway reflexes were active, the animals were extubated and put back in their cages.

\section{Post-resuscitation treatment}

After ROSC, animals were randomized to PBS-treated group $(n=24)$, MSCs-treated group $(n=24)$, topical PBS-treated group $(n=16)$, or topical TSG-6-treated group $(n=16)$. In PBS-treated or MSCs-treated group, animals received $0.5 \mathrm{~mL}$ PBS alone or $5 \times 10^{6}$ MSCs labeled with DAPI in $0.5 \mathrm{~mL}$ PBS injected into the right atrium at $2 \mathrm{~h}$ after ROSC. In topical PBS-treated or TSG-6-treated group, animals fixed in a stereotaxic apparatus (RWD, China) received $10 \mu \mathrm{L}$ PBS alone or $6 \mu \mathrm{g}$ of recombinant human TSG-6 (rhTSG-6; R\&D Systems, USA) in $10 \mu \mathrm{L}$ PBS at $2 \mathrm{~h}$ after ROSC, by injecting into the right lateral ventricle (coordinates: lateral $1.5 \mathrm{~mm}$, anterior-posterior $-0.8 \mathrm{~mm}$, 
dorsal-ventral $4 \mathrm{~mm}$ ) using a microsyringe. The injection was finished within $10 \mathrm{~min}$, and the microsyringe was left in place for 10 min after the injection and then retrieved slowly out of the brain. Neurological functional testing was evaluated at 1 , 3 , and $7 \mathrm{~d}$ after CPR by an observer blinded to experimental condition.

\section{Neurological functional testing}

Neurological deficit scores (NDS) as previously described ${ }^{[21]}$ quantitate neurological deficit on a scale of 0-80. NDS are based on a composite of arousal, reflex, motor, sensory and balance responses with 0 corresponding to brain death and 80 to no deficit.

Tape removal test (TRT) reported by Albertsmeier ${ }^{[22]}$ evaluated sensorimotor integration. The animals were trained on five times per day for 3 consecutive days before cardiac arrest. The technique included application of two small pieces of 10 $\mathrm{mm}$ by $12 \mathrm{~mm}$ adhesive tapes to both forepaws in random order. The time from attachment of adhesive tapes to the front paws until the animals completely removed them using their teeth was recorded on each of three trials lasting up to $180 \mathrm{~s}$. After training, most unimpaired animals tore off adhesive tapes within less than $20 \mathrm{~s}$.

\section{Serum S-100B concentrations}

Intravenous blood was obtained from the inferior vena cava. Blood samples were centrifuged at $310 \times g$ for $15 \mathrm{~min}$ and serum was stored at $-80^{\circ} \mathrm{C}$ until use. Serum S-100B concentrations were determined from rats at 1,3 , and $7 \mathrm{~d}$ after CPR using rat S-100B enzyme linked immunosorbent assay (ELISA) kit (CUSABIO, USA) according to the manufacturer's directions.

\section{Fluorescence evaluation of DAPI-labeled MSCs}

Three days after CPR, fluorescence from DAPI-labeled MSCs in vivo was evaluated by a fluorescent microscope (Nikon, Japan). Rats were deeply anaesthetized and sacrificed. The brains were removed, fixed in $4 \%$ paraformaldehyde in PBS and dehydrated in sequence with $20 \%$ and $30 \%$ sucrose in PBS for overnight. Continuous coronal cryostat sections $(10 \mu \mathrm{m})$ from the region between 3.5 and $3.6 \mathrm{~mm}$ posterior to Bregma were processed for fluorescence detection.

\section{ELISA analysis of proinflammatory factors in brain}

Three days after CPR, rats were reanaesthetised and euthanized. The brains were removed, and the cerebral cortex was dissected on ice and stored at $-80^{\circ} \mathrm{C}$ until use. Afterwards, $50 \mathrm{mg}$ of the cortex was placed in $500 \mu \mathrm{L}$ of cell lysate and homogenized with a Dounce homogenizer (Wheaton Science Products, Millville, USA). The homogenate was centrifuged $(13041 \times g)$ for $10 \mathrm{~min}$ at $4^{\circ} \mathrm{C}$, and the supernatant was collected and adjusted to $5 \mathrm{mg} / \mathrm{mL}$ for ELISA assay. The concentrations of interleukin (IL-1 $\beta$ and IL-6) and tumor necrosis factor- $\alpha$ (TNF- $\alpha$ ) were assayed using rat IL-1 $\beta$, IL-6, and TNF- $\alpha$ ELISA kits (CUSABIO, USA) according to the manufacturer's directions.
Real-time reverse transcription-polymerase chain reaction (RTPCR) analysis

Three days after CPR, the brains of anaesthetized rats were removed, and the cerebral cortex was collected and stored at $-80^{\circ} \mathrm{C}$. Total RNA was extracted from the cerebral cortex using TRIZOL reagent (Invitrogen, USA) according to the manufacturer's directions. Reverse transcription was performed using PrimeScript RT Master Mix (TaKaRa, China). The cDNA amplification was performed by real-time PCR (LightCycler 480, Roche, USA) using GoTaq qPCR Master Mix (Promega, USA). RT-PCR primers were designed by Beacon 7.0 Primer Express software (Applied Biosystems, Carlsbad, USA). Specific primers of TSG-6, IL-1 $\beta$, IL-6, TNF- $\alpha$, and glyceraldehyde3 -phosphate dehydrogenase $(\mathrm{GAPDH})$ in this study were listed as follows: TSG-6 forward, 5'-AAGCAGCCAGAAAGATTGGA-3'; reverse, 5'-TTCGGGTTGTAGCAATAGGC-3'; IL-1 $\beta$ forward, 5'-TCCTCTGTGACTCGTG GGAT-3'; reverse, 5'-TCAGACAGCACGAGGCATTT-3'; IL-6 forward, 5'-AGAGACTTCCAGCCAGTTGC-3'; reverse, 5' -AGCCTCCGACTTGTGAAGTG-3'; TNF-a forward, 5'-TCGTCTACTCCTCAGAGCCC-3'; reverse, 5'-ACTTCAGCGTCTCGTGTGTT-3'; GAPDH forward, 5'-CAAGGTCATCCATGACAACTTTG-3'; reverse, 5'-GTCCACCACCCTGTTGCTGTAG-3'. Real-time PCR reactions were incubated at $95^{\circ} \mathrm{C}$ for $2 \mathrm{~min}$, and then 40 cycles at $95^{\circ} \mathrm{C}$ for $3 \mathrm{~s}$ followed by $60^{\circ} \mathrm{C}$ for $30 \mathrm{~s}$. Relative mRNA expression levels of the target genes were calculated based on the $2^{-\Delta \Delta / \mathrm{Cts}}$ methods normalized to mRNA levels for GAPDH.

\section{Western blotting analysis}

Expression of TSG-6 and neutrophil elastase (NE) in the cerebral cortex at $3 \mathrm{~d}$ after CPR was analyzed by Western blotting. Aliquots of proteins from cortical supernatants were separated by sodium dodecyl sulfate-polyacrylamide gel electrophoresis (SDS-PAGE) using 10\% acrylamide gels. The proteins were electrotransferred onto PVDF membranes, and the membranes were blocked with 5\% skim milk in Tris buffered saline with $0.1 \%$ Tween 20 (TBST). Blotting membranes were incubated with corresponding primary antibodies containing goat antiTSG-6 (1:800, Santa Cruz, USA), rabbit anti-NE (1:500, Abcam, USA), or mouse anti-GAPDH (1:5000, Santa Cruz, USA) at $4{ }^{\circ} \mathrm{C}$ overnight. The membranes were washed with TBST and incubated with the appropriate secondary antibody for $1 \mathrm{~h}$ at room temperature. The protein bands on the membranes were detected by chemiluminescence, scanned and analyzed by an image analyzer (BandScan 5.0). Protein levels were expressed as a ratio relative to GAPDH in the same sample.

\section{Statistical analysis}

SPSS 13.0 software was used for statistical analysis. Normally distributed variables are presented as mean $\pm S D$, and nonnormally distributed variables as median (25th, 75th percentile). Multiple comparisons were made by one-way ANOVA or Kruskal-Wallis $\mathrm{H}$ test, followed by Bonferronni' post hoc test. Data between two groups of each measurement time were compared by analysis of variance for repeated measures. 
$P$ values of less than 0.05 were considered statistically significant.

\section{Results}

\section{Baseline physiologic variables}

There were no significant differences in baseline physiologic parameters among the five groups before inducing cardiac arrest (Table 1).

\section{MSCs treatment improved neurological outcome}

All sham-operated animals had normal NDS at all time points (NDS 80). Differences in NDS between PBS-treated and MSCstreated groups were not observed at $1 \mathrm{~d}$ after CPR. However, MSCs-treated group demonstrated significantly higher NDS than PBS-treated group at 3 and $7 \mathrm{~d}$ after CPR, indicating better neurological outcome (Figure 1A).

Before cardiac arrest, all animals tore off the tapes rapidly. After CPR, all animals had an obvious sensorimotor deficit on all testing days until $7 \mathrm{~d}$. There were no significant differences in time needed for tape removal between PBS-treated and MSCs-treated groups at $1 \mathrm{~d}$ after CPR. However, MSCstreated group needed significantly less time to remove the adhesive tapes than PBS-treated group at 3 and $7 \mathrm{~d}$ after CPR (Figure 1B).

\section{MSCs treatment reduced serum S-100B levels}

Serum S-100B levels in sham rats were very low and sharply increased after CPR. Although serum S-100B levels in MSCstreated group were not significantly different from those of PBS-treated group at $1 \mathrm{~d}$ after CPR, serum S-100B levels were significantly reduced in MSCs-treated group at 3 and $7 \mathrm{~d}$ after CPR compared with PBS-treated group, indicating less brain damage (Figure 2).

\section{Distribution of donor MSCs in damaged brain}

Three days after DAPI-labeled MSCs had been intravenously administered into cardiac arrest rats, DAPI-labeled cells were primarily observed in the cerebral cortex and had an irregular distribution (Figure 3A). However, there were very few DAPI-labeled cells observed in the hippocampus (Figure 3B).
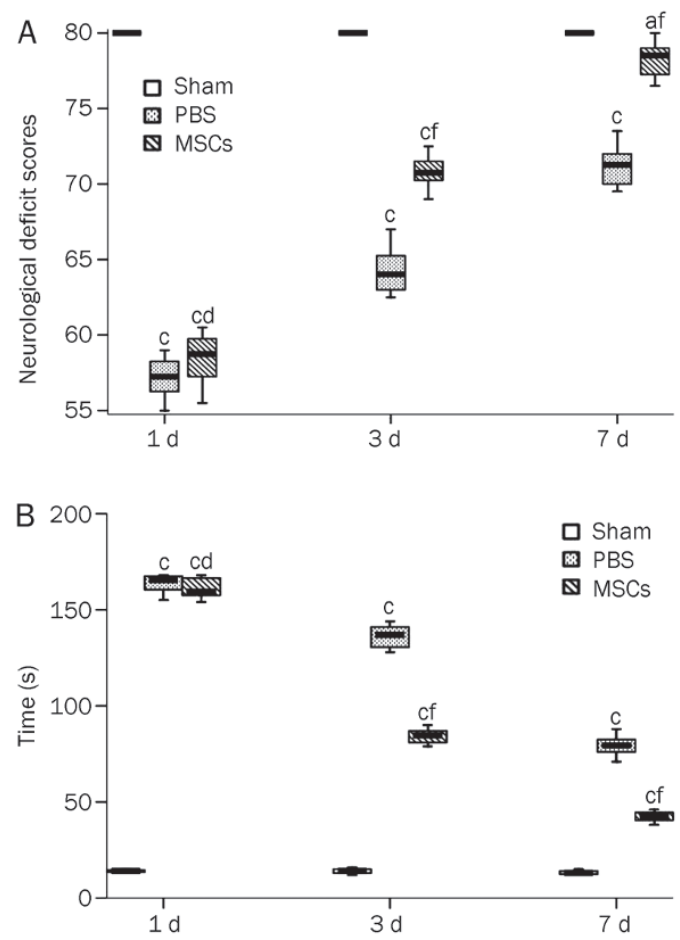

Figure 1. Neurological functional testing. (A) Neurological deficit scores test for sham, PBS-treated, and MSCs-treated rats at 1, 3, and $7 \mathrm{~d}$ after CPR. (B) Tape removal test for sham, PBS-treated, and MSCs-treated rats at 1,3 , and $7 \mathrm{~d}$ after CPR. Mean \pm SD. $n=5-8$ /group. ${ }^{\mathrm{a}} P>0.05,{ }^{\mathrm{c}} P<0.01$ vs sham rats; ${ }^{\mathrm{d}} P>0.05,{ }^{\mathrm{f}} P<0.01$ vs PBS-treated rats. PBS, phosphate buffered solution; MSCs, mesenchymal stem cells; CPR, cardiopulmonary resuscitation.

\section{MSCs treatment increased brain TSG-6 expression}

At $3 \mathrm{~d}$ after CPR, TSG-6 mRNA and protein in the cerebral cortex were assayed by real-time RT-PCR and Western blot, respectively. The results showed TSG-6 mRNA levels were very low in sham group. Global cerebral ischemia increased TSG-6 mRNA expression compared with sham group. With MSCs treatment, TSG-6 mRNA levels further increased compared with those in PBS-treated group (Figure 4A). Similar

Table 1. Baseline variables among the five groups before cardiac arrest.

\begin{tabular}{|c|c|c|c|c|c|}
\hline Variables & $\begin{array}{l}\text { Sham } \\
(n=15)\end{array}$ & $\begin{array}{c}\text { PBS } \\
(n=24)\end{array}$ & $\begin{array}{l}\text { MSCs } \\
(n=24)\end{array}$ & $\begin{array}{c}\text { Topical PBS } \\
\quad(n=16)\end{array}$ & $\begin{array}{c}\text { Topical TSG-6 } \\
\quad(n=16)\end{array}$ \\
\hline Body weight (g) & $361 \pm 22$ & $339 \pm 37$ & $328 \pm 12$ & $349 \pm 33$ & $337 \pm 12$ \\
\hline Rectal temperature $\left({ }^{\circ} \mathrm{C}\right)$ & $36.2 \pm 0.2$ & $36.4 \pm 0.1$ & $36.7 \pm 0.5$ & $36.5 \pm 0.4$ & $36.3 \pm 0.1$ \\
\hline MAP (mmHg) & $137 \pm 2.4$ & $132 \pm 8.5$ & $131 \pm 11.1$ & $133 \pm 6.6$ & $130 \pm 12.2$ \\
\hline $\mathrm{pH}$ & $7.406 \pm 0.032$ & $7.411 \pm 0.028$ & $7.387 \pm 0.043$ & $7.396 \pm 0.019$ & $7.402 \pm 0.024$ \\
\hline Lactic acid (mmol/L) & $0.7 \pm 0.04$ & $0.5 \pm 0.02$ & $0.8 \pm 0.05$ & $0.5 \pm 0.04$ & $0.6 \pm 0.06$ \\
\hline
\end{tabular}

PBS, phosphate buffered solution; MSCs, mesenchymal stem cells; MAP, mean arterial pressure; TSG-6, tumor necrosis factor- $\alpha$-induced protein 6 . 


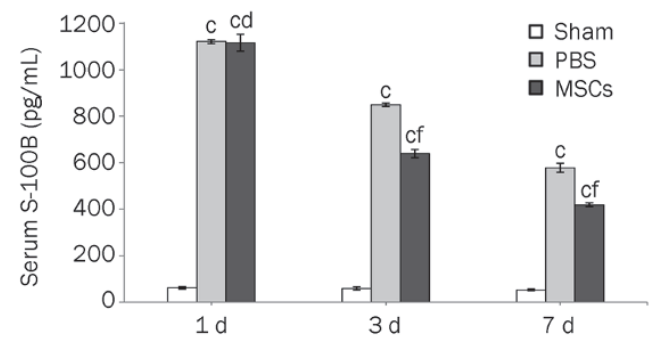

Figure 2. Serum S-100B levels determined by S-100B ELISA in sham, PBS-treated, and MSCs-treated rats at 1, 3, and $7 \mathrm{~d}$ after CPR. MSCs treatment significantly reduced serum S-100B levels at 3 and $7 \mathrm{~d}$ after CPR. Mean \pm SD. $n=5-8$ at every separate time point for each group. ${ }^{\mathrm{c}} P<0.01$ vs sham rats. ${ }^{\mathrm{d}} P>0.05,{ }^{\mathrm{f}} P<0.01$ vs $\mathrm{PBS}$-treated rats. $\mathrm{PBS}$, phosphate buffered solution; MSCs, mesenchymal stem cells; CPR, cardiopulmonary resuscitation.
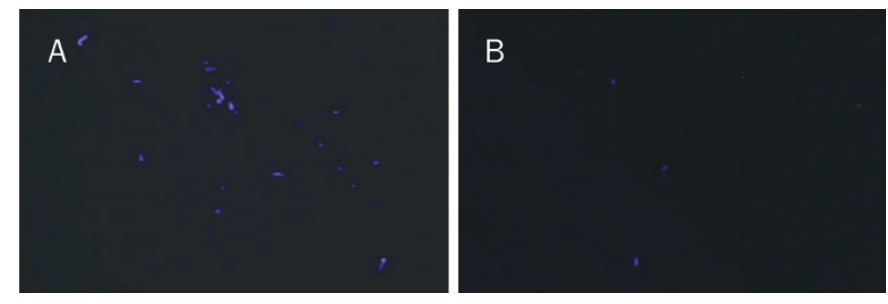

Figure 3. MSCs in damaged brain under fluorescent microscope at $3 \mathrm{~d}$ after intravenously administered DAPI-labeled MSCs in MSCs-treated rats. DAPI-labeled cells (Blue round or oval nucleus) were primarily present in the cerebral cortex $(A)$ and there were very few DAPI-labeled cells in the hippocampus (B). $n=5$. Magnification: $\times 200$.

results were observed by Western blot for TSG- 6 protein at $3 \mathrm{~d}$ after CPR (Figure 4B, 4C).

\section{MSCs treatment reduced brain neutrophil elastase expression}

At $3 \mathrm{~d}$ after CPR, Western blot analysis of NE protein in the cerebral cortex for PBS-treated group revealed significant increases compared with sham group, suggesting the involvement of NE in cortical inflammation. However, intravenously administered MSCs significantly reduced NE levels compared with PBS-treated group (Figure 5).

\section{MSCs treatment decreased brain proinflammatory factors expression}

After CPR from cardiac arrest, proinflammatory factors (IL-1 $\beta$, IL-6, and TNF-a) may play an important role in ischemic brain damage. These cytokines in the cerebral cortex were assayed by real-time RT-PCR and ELISA at $3 \mathrm{~d}$ after CPR. The results showed the expression of IL-1 $\beta$, IL-6, and TNF- $\alpha$ mRNA in the cerebral cortex for PBS-treated rats was significantly increased compared with sham rats. However, MSCs treatment significantly decreased these cytokines expression compared with PBS-treated rats (Figure 6A). Similar results were observed by ELISA for IL-1 $\beta$, IL-6, and TNF- $\alpha$ at $3 \mathrm{~d}$ after CPR (Figure 6B).
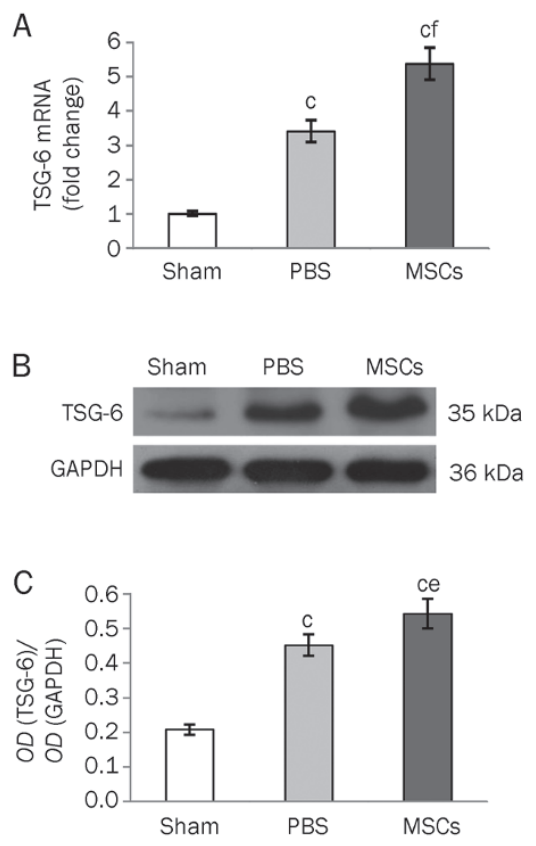

Figure 4. Tumor necrosis factor- $\alpha$-induced protein 6 (TSG-6) expression in the cerebral cortex at $3 d$ after CPR from cardiac arrest rats. (A) Real-time RT-PCR assays for TSG- 6 in sham, PBS-treated, and MSCs-treated groups. (B) Western blot analysis of TSG-6 protein. (C) Densitometry analysis of TSG-6. Mean \pm SD. $n=5-8$ /group. ${ }^{\mathrm{C}} P<0.01$ vs sham group. ${ }^{\mathrm{e}} P<0.05$, ${ }^{f} P<0.01$ vs PBS-treated group. PBS, phosphate buffer solution; MSCs, mesenchymal stem cells; CPR, cardiopulmonary resuscitation.

A
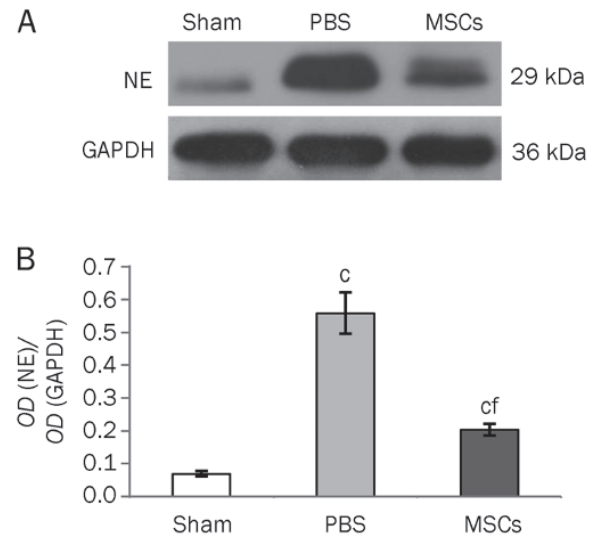

Figure 5. Neutrophil elastase (NE) expression in the cerebral cortex at $3 \mathrm{~d}$ after CPR from cardiac arrest rats. (A) Western blot analysis of NE protein in sham, PBS-treated, and MSCs-treated groups. (B) Densitometry analysis of NE. Mean \pm SD. $n=5-8$ /group. ${ }^{c} P<0.01$ vs sham group. ${ }^{\mathrm{f}} P<0.01$ vs PBS-treated group. PBS, phosphate buffer solution; MSCs, mesenchymal stem cells; CPR, cardiopulmonary resuscitation.

Administration of TSG- 6 into the lateral ventricle reduced brain inflammation and improved neurological outcome

Topical application of rhTSG-6 also significantly decreased NE and proinflammatory factors expression and promoted functional recovery compared with topical PBS-treated rats, which 
A

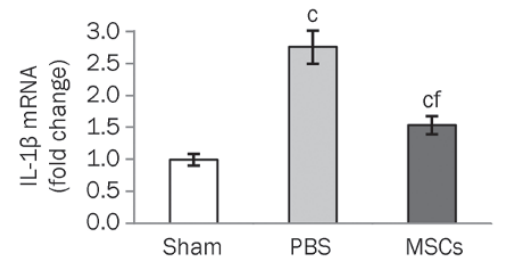

B

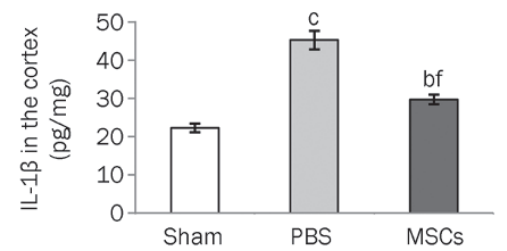

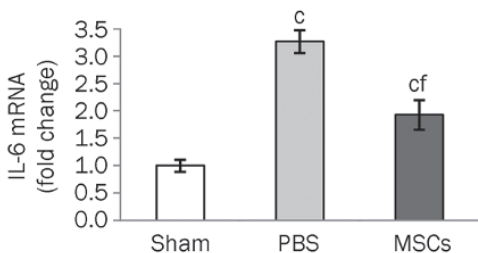

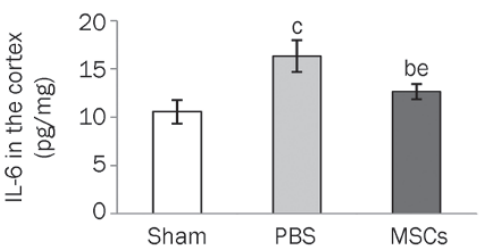

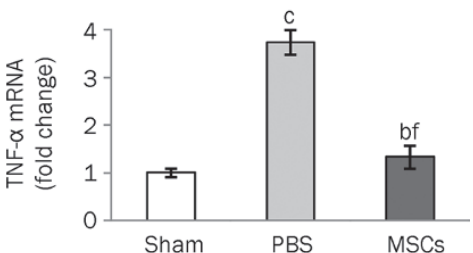

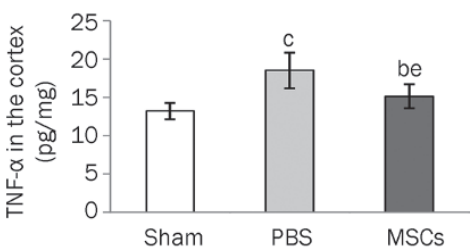

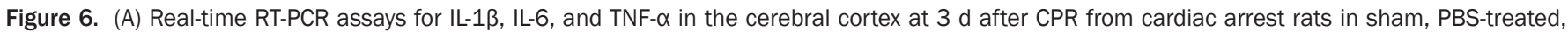

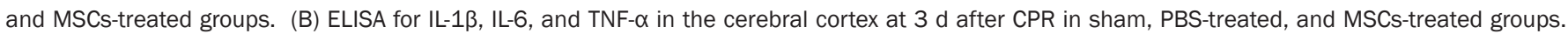

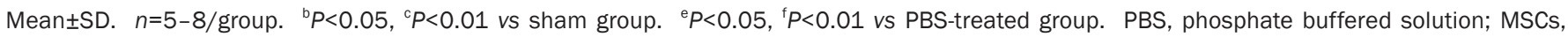
mesenchymal stem cells; CPR, cardiopulmonary resuscitation.

reproduced the therapeutic effects of intravenously administered MSCs (Figure 7).

\section{Discussion}

The present study demonstrated that intravenously administered MSCs after global cerebral ischemia induced by
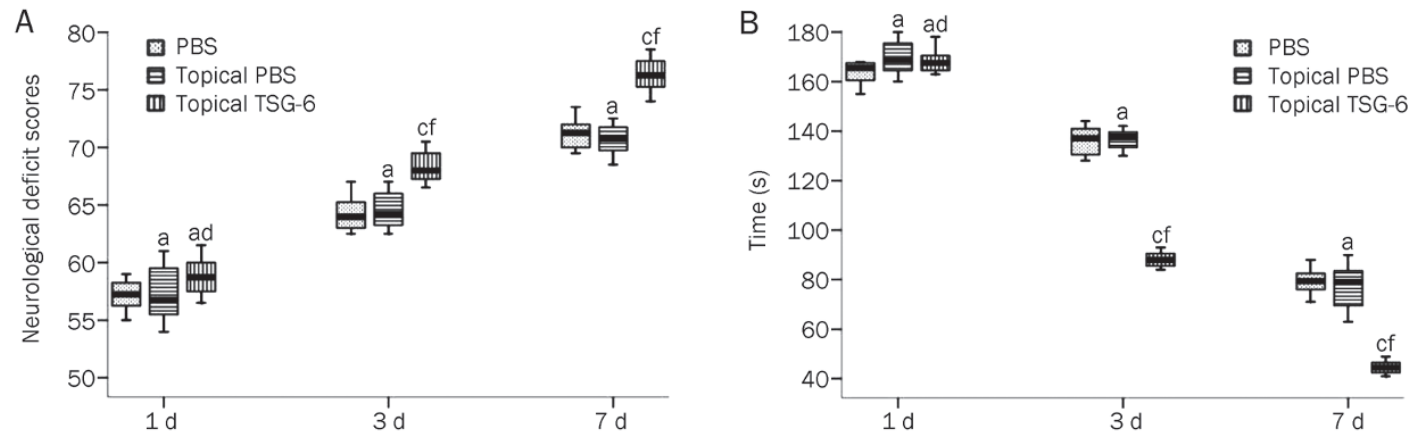

C
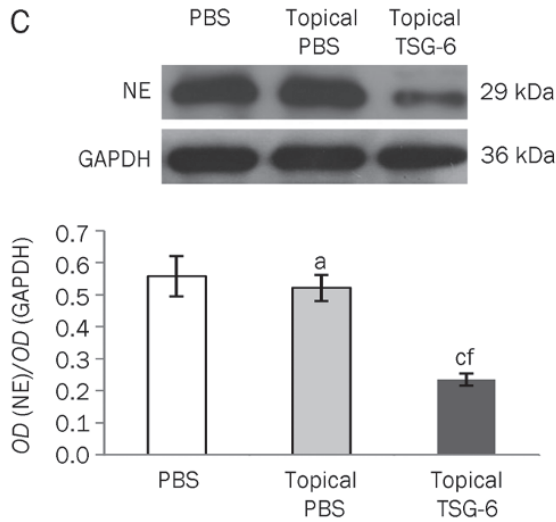

D

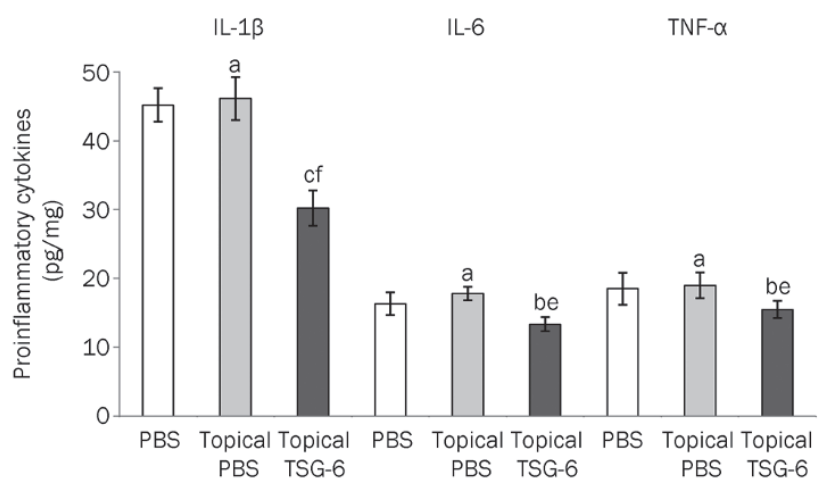

Figure 7. Topical application of TSG-6 reproduced the therapeutic effects of intravenously administered MSCs. (A) Neurological deficit scores test for PBS-treated, topical PBS-treated, and TSG-6-treated rats at 1, 3, and $7 \mathrm{~d}$ after CPR. (B) Tape removal test for PBS-treated, topical PBS-treated, and TSG6-treated rats at 1,3 , and $7 \mathrm{~d}$ after CPR. (C) Western blot for NE expression in the cerebral cortex at $3 \mathrm{~d}$ after CPR. (D) ELISA for IL-1 $\beta$, IL-6, and TNF- $\alpha$ in the cerebral cortex at $3 \mathrm{~d}$ after CPR. Mean \pm SD. $n=8$ /group. ${ }^{\mathrm{a}} P>0.05,{ }^{\mathrm{b}} P<0.05,{ }^{\mathrm{c}} P<0.01$ vs PBS-treated rats. ${ }^{\mathrm{d}} P>0.05,{ }^{\mathrm{e}} P<0.05,{ }^{\mathrm{f}} P<0.01$ vs topical PBS-treated rats. MSCs, mesenchymal stem cells; PBS, phosphate buffer solution; CPR, cardiopulmonary resuscitation; TSG-6, tumor necrosis factor- $\alpha$ induced protein 6; NE, neutrophil elastase. 
cardiac arrest reduced brain inflammatory responses and improved neurological function. Also, the results suggested that MSCs treatment up-regulated TSG-6 in the cerebral cortex and administration of rhTSG-6 into the lateral ventricle reproduced the therapeutic effects of MSCs on inflammatory damage to the brain, indicating that TSG-6 may suppress the excessive inflammatory responses to global cerebral ischemia and contribute to neurological functional recovery after MSCs treatment. Although the present study was not designed to investigate mechanisms of how MSCs up-regulated TSG-6, possible mechanisms of TSG-6 increase after MSCs treatment include: MSCs either stimulate endogenous brain tissues to secret TSG-6 or directly secret TSG-6.

The pathophysiologic mechanisms of brain damage caused by cardiac arrest and CPR include complete temporary global cerebral ischemia and secondary inflammatory responses during and after $\mathrm{CPR}^{[5]}$. A number of studies reported that postischemic inflammatory responses marked by increased levels of brain tissue NE or proinflammatory factors (IL-1 $\beta$, IL-6, and TNF- $\alpha$ ) were involved in brain damage after cerebral ischemia $^{[23-26]}$. Pharmacologic inhibition of NE or proinflammatory factors following cerebral ischemia protected against neuronal damages and neurological deficits ${ }^{[23,24,27,28]}$. A recent study showed that treatment with rat umbilical cord matrix cells modulated the inflammatory response to global cerebral ischemia and protected against neuronal injury after global cerebral ischemia ${ }^{[29]}$. Consistent with the above researches, the results here demonstrated that MSCs treatment offered protection against brain damage after global cerebral ischemia by suppressing the up-regulation of brain tissue NE and proinflammatory cytokines (IL-1 $\beta$, IL-6, and TNF- $\alpha$ ).

Although the present study showed that MSCs treatment suppressed inflammatory responses to global cerebral ischemia, the mechanisms of how MSCs down-regulated NE and proinflammatory cytokines were not clarified. In recent years, a multifunctional glycoprotein TSG-6 is of major interest because the growing amount of evidence has shown that transplanted MSCs secrete TSG-6 in vivo in response to inflammatory conditions including cornea injury ${ }^{[30]}$, lung injury ${ }^{[31]}$, myocardial infarction ${ }^{[32]}$, and peritonitis ${ }^{[33]}$. This led us to investigate TSG-6 expression levels of the brain in global cerebral ischemic rats following systematically administered MSCs. The results showed that MSCs treatment significantly increased the levels of brain tissue TSG-6, suggesting the TSG-6 may improve neurological function after MSCs treatment. Increased TSG-6 protein could bind to the fragments of hyaluronan and exert strong anti-inflammatory effects ${ }^{[34,35]}$.

TSG-6 is a $35 \mathrm{kDa}$ secreted protein composed mainly of contiguous Link and CUB modules ${ }^{[35]}$. The protein is little or not expressed in adult tissues, but is synthesized by MSCs and many other cell types in response to stimulation with TNF-a and other proinflammatory cytokines ${ }^{[35]}$. The experimental studies from several murine models demonstrated that administration of the recombinant protein improved arthritis ${ }^{[36,37]}$ and suppressed the excessive inflammatory responses to the corneal injury ${ }^{[30,38]}$, lung injury ${ }^{[31]}$, and myocardial infarction ${ }^{[32]}$.
So we assumed that the same anti-inflammatory activities might be involved in global cerebral ischemia. To test the hypothesis that TSG-6 can decrease inflammatory responses and improve neurological function, rhTSG-6 was injected into the lateral ventricle $2 \mathrm{~h}$ after CPR from cardiac arrest rats. The results showed that topical application of rhTSG-6 also reduced brain inflammatory responses and improved neurological function, suggesting that rhTSG-6 reproduced the therapeutic effects of MSCs on inflammatory responses and neurological deficit. Combined, MSCs treatment achieved a neuroprotective effect after global cerebral ischemia in rats possibly via the elevated TSG-6.

A pathological study showed that the prelabeled transplanted MSCs were found primarily in the cerebral cortex which was particularly affected by ischemia, suggesting intravenously injected MSCs crossed the blood-brain barrier and migrated towards injured tissue ${ }^{[6,14]}$. After transplantation into injured tissue, what's the fate of MSCs in the cortex? One possibility is that transplanted MSCs could differentiate into cells of neural lineage and replace the damaged tissue ${ }^{[7]}$. However, the explanation is challenged by recent observations suggesting that only small numbers of MSCs engraft into injured tissue $^{[32]}$. A far more reasonable explanation is that therapeutic effects of MSCs are likely to depend on paracrine mechanisms mediated by the release of growth factors, anti-apoptotic factors and anti-inflammatory cytokines ${ }^{[39]}$. These inconsistencies suggest that more investigation is required to identify the cellular fate of transplanted MSCs and elucidate the mechanisms by which MSCs promote functional recovery.

However, the study also suffers from several limitations. One limitation is that the study does not explicitly show that TSG-6 is a factor in improving functional recovery after treatment of global cerebral ischemia with MSCs. Although recent reports have shown that MSCs treatment significantly reduces inflammatory responses by secretion of TSG-6 in several animal models of inflammation ${ }^{[33-36]}$, the possibility that endogenous brain tissues secret TSG-6 can not be completely excluded. The further research performed is to block TSG-6 in rats using TSG-6 antibody or in vivo siRNA methodology. Another limitation is that the mechanisms of how TSG-6 suppresses inflammatory responses are not elucidated. Possible anti-inflammatory mechanisms of TSG-6 include ${ }^{[34,35]}$ : (1) It down-regulates the protease network by increasing antiplasmin activity of inter-a-inhibitor; (2) It inhibits neutrophil migration into sites of inflammation; (3) It can regulate the expression of various factors such as cyclo-oxygenase-2 (COX-2) that control inflammatory responses. Of course, its precise mechanism of action remains to be elucidated.

In conclusion, systemically administered MSCs reduced inflammatory responses to global cerebral ischemia and improved neurological function in rats possibly via the elevated TSG-6.

\section{Acknowledgements}

This study was supported in part by a research grant from National Natural Science Foundation of China (81272061) and 
the Fundamental Research Funds for the Central Universities and Yat-Sen Scholarship for Young Scientists.

We thank Xing-yong CHEN for his assistance with pathological examination.

\section{Author contribution}

Xiang-shao FANG, Yue FU, and Zi-tong HUANG designed the research. Qing-ming LIN, Shen ZHAO, and Li-li ZHOU performed the research. Qing-ming LIN, Shen ZHAO, and Zitong HUANG analyzed the data and wrote the paper.

\section{References}

1 Jones AE. Hypothermia after cardiac arrest: we can do this. Acad Emerg Med 2008; 15: 558-9.

2 Arrich J, Holzer M, Havel C, Müllner M, Herkner H. Hypothermia for neuroprotection in adults after cardiopulmonary resuscitation. Cochrane Database Syst Rev 2012; 9: CD004128.

3 Graves JR, Herlitz J, Bång A, Axelsson A, Ekström L, Holmberg M, et al. Survivors of out of hospital cardiac arrest: their prognosis, longevity and functional status. Resuscitation 1997; 35: 117-21.

4 Vaagenes P, Safar P, Moossy J, Rao G, Diven W, Ravi C, et al. Asphyxiation versus ventricular fibrillation cardiac arrest in dogs. Differences in cerebral resuscitation effects - a preliminary study. Resuscitation 1997; 35: 41-52.

5 Xiao F. Bench to bedside: brain edema and cerebral resuscitation: the present and future. Acad Emerg Med 2002; 9: 933-46.

6 Wang T, Tang W, Sun S, Xu T, Wang H, Guan J, et al. Intravenous infusion of bone marrow mesenchymal stem cells improves brain function after resuscitation from cardiac arrest. Crit Care Med 2008; 36: 486-91.

7 Parr AM, Tator $\mathrm{CH}$, Keating A. Bone marrow-derived mesenchymal stromal cells for the repair of central nervous system injury. Bone Marrow Transplant 2007; 40: 609-19.

8 Toyama K, Honmou O, Harada K, Suzuki J, Houkin K, Hamada H, et al. Therapeutic benefits of angiogenetic gene-modified human mesenchymal stem cells after cerebral ischemia. Exp Neurol 2009; 216: $47-55$.

9 Onda T, Honmou O, Harada K, Houkin K, Hamada H, Kocsis JD. Therapeutic benefits by human mesenchymal stem cells (hMSCs) and Ang-1 gene-modified hMSCs after cerebral ischemia. J Cereb Blood Flow Metab 2008; 28: 329-40.

10 Omori Y, Honmou O, Harada K, Suzuki J, Houkin K, Kocsis JD. Optimization of a therapeutic protocol for intravenous injection of human mesenchymal stem cells after cerebral ischemia in adult rats. Brain Res 2008; 1236: 30-8.

11 Komatsu K, Honmou O, Suzuki J, Houkin K, Hamada H, Kocsis JD. Therapeutic time window of mesenchymal stem cells derived from bone marrow after cerebral ischemia. Brain Res 2010; 1334: 84-92.

12 Horita Y, Honmou O, Harada K, Houkin K, Hamada H, Kocsis JD. Intravenous administration of glial cell line-derived neurotrophic factor gene-modified human mesenchymal stem cells protects against injury in a cerebral ischemia model in the adult rat. J Neurosci Res 2006; 84: 1495-504.

13 Wakabayashi K, Nagai A, Sheikh AM, Shiota Y, Narantuya D, Watanabe $\mathrm{T}$, et al. Transplantation of human mesenchymal stem cells promotes functional improvement and increased expression of neurotrophic factors in a rat focal cerebral ischemia model. J Neurosci Res 2010; 88: 1017-25.

14 Zheng W, Honmou O, Miyata K, Harada K, Suzuki J, Liu H, et al. Therapeutic benefits of human mesenchymal stem cells derived from bone marrow after global cerebral ischemia. Brain Res 2010; 1310: 8-16.

15 Ohtaki H, Ylostalo JH, Foraker JE, Robinson AP, Reger RL, Shioda S, et al. Stem/progenitor cells from bone marrow decrease neuronal death in global ischemia by modulation of inflammatory/immune responses. Proc Natl Acad Sci U S A 2008; 105: 14638-43.

16 Zhang J, Li Y, Chen J, Yang M, Katakowski M, Lu M, et al. Expression of insulin-like growth factor 1 and receptor in ischemic rats treated with human marrow stromal cells. Brain Res 2004; 1030: 19-27.

17 Chen J, Li Y, Zhang R, Katakowski M, Gautam SC, Xu Y, et al. Combination therapy of stroke in rats with a nitric oxide donor and human bone marrow stromal cells enhances angiogenesis and neurogenesis. Brain Res 2004; 1005: 21-8.

18 Wang T, Tang W, Sun S, Wan Z, Xu T, Huang Z, et al. Mesenchymal stem cells improve outcomes of cardiopulmonary resuscitation in myocardial infarcted rats. J Mol Cell Cardiol 2009; 46: 378-84.

19 Castanheira P, Torquetti LT, Magalhãs DR, Nehemy MB, Goes AM. DAPI diffusion after intravitreal injection of mesenchymal stem cells in the injured retina of rats. Cell Transplant 2009; 18: 423-31.

20 Katz LM, Wang Y, Rockoff S, Bouldin TW. Low-dose Carbicarb improves cerebral outcome after asphyxial cardiac arrest in rats. Ann Emerg Med 2002; 39: 359-65.

21 Geocadin RG, Ghodadra R, Kimura T, Lei H, Sherman DL, Hanley $\mathrm{DF}$, et al. A novel quantitative EEG injury measure of global cerebral ischemia. Clin Neurophysiol 2000; 111: 1779-87.

22 Albertsmeier M, Teschendorf P, Popp E, Galmbacher R, Vogel P, Böttiger BW. Evaluation of a tape removal test to assess neurological deficit after cardiac arrest in rats. Resuscitation 2007; 74: 552-8.

23 He ZJ, Huang ZT, Chen XT, Zou ZJ. Effects of matrix metalloproteinase 9 inhibition on the blood brain barrier and inflammation in rats following cardiopulmonary resuscitation. Chin Med J 2009; 122: 2346-51.

24 Xuan A, Long D, Li J, Ji W, Hong L, Zhang M, et al. Neuroprotective effects of valproic acid following transient global ischemia in rats. Life Sci 2012; 90: 463-8.

25 Orzyłowska O, Oderfeld-Nowak B, Zaremba M, Januszewski S, Mossakowski M. Prolonged and concomitant induction of astroglial immunoreactivity of interleukin-1beta and interleukin- 6 in the rat hippocampus after transient global ischemia. Neurosci Lett 1999; 263: 72-6.

26 Stowe AM, Adair-Kirk TL, Gonzales ER, Perez RS, Shah AR, Park TS, et al. Neutrophil elastase and neurovascular injury following focal stroke and reperfusion. Neurobiol Dis 2009; 35: 82-90.

27 Matayoshi H, Hirata T, Yamashita S, Ishida K, Mizukami Y, Gondo T, et al. Neutrophil elastase inhibitor attenuates hippocampal neuronal damage after transient forebrain ischemia in rats. Brain Res 2009; 1259: 98-106.

28 Shimakura A, Kamanaka Y, Ikeda Y, Kondo K, Suzuki Y, Umemura K. Neutrophil elastase inhibition reduces cerebral ischemic damage in the middle cerebral artery occlusion. Brain Res 2000; 858: 55-60.

29 Hirko AC, Dallasen R, Jomura S, Xu Y. Modulation of inflammatory responses after global ischemia by transplanted umbilical cord matrix stem cells. Stem Cells 2008; 26: 2893-901.

30 Roddy GW, Oh JY, Lee RH, Bartosh TJ, Ylostalo J, Coble K, et al. Action at a distance: systemically administered adult stem/progenitor cells (MSCs) reduce inflammatory damage to the cornea without engraftment and primarily by secretion of TNF- $\alpha$ stimulated gene/protein 6 . Stem Cells 2011; 29: 1572-9.

31 Danchuk S, Ylostalo JH, Hossain F, Sorge R, Ramsey A, Bonvillain RW, et al. Human multipotent stromal cells attenuate lipopolysaccharideinduced acute lung injury in mice via secretion of tumor necrosis factor- $\alpha$-induced protein 6. Stem Cell Res Ther 2011; 2: 27. 
32 Lee RH, Pulin AA, Seo MJ, Kota DJ, Ylostalo J, Larson BL, et al. Intravenous hMSCs improve myocardial infarction in mice because cells embolized in lung are activated to secrete the anti-inflammatory protein TSG-6. Cell Stem Cell 2009; 5: 54-63.

33 Choi H, Lee RH, Bazhanov N, Oh JY, Prockop DJ. Anti-inflammatory protein TSG- 6 secreted by activated MSCs attenuates zymosaninduced mouse peritonitis by decreasing TLR2/NF-KB signaling in resident macrophages. Blood 2011; 118: 330-8.

34 Getting SJ, Mahoney DJ, Cao T, Rugg MS, Fries E, Milner CM, et al. The link module from human TSG-6 inhibits neutrophil migration in a hyaluronan- and inter-alpha-inhibitor-independent manner. J Biol Chem 2002; 277: 51068-76.

35 Milner CM, Higman VA, Day AJ. TSG-6: a pluripotent inflammatory mediator? Biochem Soc Trans 2006; 34: 446-50.

36 Bárdos T, Kamath RV, Mikecz K, Glant TT. Anti-inflammatory and chondroprotective effect of TSG-6 (tumor necrosis factor-alphastimulated gene-6) in murine models of experimental arthritis. Am J Pathol 2001; 159: 1711-21.

37 Mindrescu C, Thorbecke GJ, Klein MJ, Vilcek J, Wisniewski HG. Amelioration of collagen-induced arthritis in DBA/1J mice by recombinant TSG-6, a tumor necrosis factor/interleukin-1-inducible protein. Arthritis Rheum 2000; 43: 2668-77.

38 Oh JY, Roddy GW, Choi H, Lee RH, Ylöstalo JH, Rosa RH Jr, et al. Antiinflammatory protein TSG- 6 reduces inflammatory damage to the cornea following chemical and mechanical injury. Proc Natl Acad Sci U S A 2010; 107: 16875-80.

39 Uccelli A, Benvenuto F, Laroni A, Giunti D. Neuroprotective features of mesenchymal stem cells. Best Pract Res Clin Haematol 2011; 24 : 59-64. 